\title{
ESTIMATING COMPLEX EIGENVALUES OF NON-SELF ADJOINT SCHRÖDINGER OPERATORS VIA COMPLEX DILATIONS
}

\author{
JefFrey H. SCHENKER
}

\begin{abstract}
The phenomenon "hypo-coercivity," i.e., the increased rate of contraction for a semi-group upon adding a large skew-adjoint part to the generator, is considered for $1 \mathrm{D}$ semi-groups generated by the Schrödinger operators $-\partial_{x}^{2}+x^{2}+\mathrm{i} \gamma f(x)$ with a complex potential. For $f$ of the special form $f(x)=1 /\left(1+|x|^{\kappa}\right)$, it is shown using complex dilations that the real part of eigenvalues of the operator are larger than a constant times $|\gamma|^{2 /(\kappa+2)}$.
\end{abstract}

\section{Introduction}

The purpose of this note is to take up the following problem suggested by Villani [9] in the proceedings of the last ICM, quoted here (with notation slightly changed):

Identify sufficient conditions on $f: \mathbb{R} \rightarrow \mathbb{R}$, so that the real parts of the eigenvalues of

$$
L_{\gamma}: \psi \mapsto\left(-\partial_{x}^{2} \psi+x^{2} \psi\right)+\mathrm{i} \gamma f \psi,
$$

as on operator on $L^{2}$ go to infinity as $|\gamma| \rightarrow \infty$ and estimate the rate of divergence.

The stated problem is a model for the phenomenon of "hypocoercivity," with the specific form motivated by applications described in [9] and outlined briefly below.

It turns out that a sufficient condition on $f$ for divergence of the real parts of the eigenvalues may be obtained using a general result due to Constantin et al. [1, Theorem 1.4]. Specifically, we will show below that:

Theorem 1.1. The real parts of the eigenvalues of $L_{\gamma}$ diverge to $\infty$ as $\gamma \rightarrow \infty$ if $\{x: f(x)=t\}$ is essentially nowhere dense for each $t \in \mathbb{R}$.

Remarks. (1) A set $S$ is essentially nowhere dense if $S=S^{\prime} \cup N$ where $S^{\prime}$ is no-where dense and $N$ has Lebesgue measure zero. (2) Since the operators $L_{\gamma}$ are unbounded, we should specify their domains. Throughout we will consider bounded $f$, so the natural choice is to consider $L_{\gamma}$ on the domain of self-adjointness for the real part $L_{0}$ :

$$
\mathcal{D}=\left\{\psi: \int\left|\partial_{x} \psi\right|^{2}+x^{2}|\psi|^{2}<\infty\right\} .
$$

It is then not hard to show that $L_{\gamma}$ is the generator of a contractive semi-group and that $L_{\gamma}^{-1}$ is compact. Thus the spectrum $\sigma\left(L_{\gamma}\right)$ consists only of eigenvalues. (3) In fact the "if" of the theorem could be replaced by an "if and only if" [10] - a proof of

Received by the editors August 2, 2010. 
the reverse implication would follow, for example, from the method used recently to derive a similar result in the context of diffusion with drift on a compact manifold [3].

However, the question of estimating the rate of divergence seems to be much more delicate. In particular, it is likely that the rate will depend on the specific form of $f$ and thus we may not expect such a general result. Nonetheless, as we will show below, a number of interesting examples with analytic $f$ may be analyzed with the technique of complex dilations, yielding, in one case at least, a very good estimate on the rate.

The method of complex dilations, as applied below, essentially requires that $f$ be analytic, possibly with a branch point. Furthermore, as the proof will show, the asymptotic growth of eigenvalues depends essentially on $f$ only in the neighborhood of a critical point. If the potential has multiple critical points one may expect each of these to contribute. For the sake of clarity, we will not try to formulate a very general result, but rather focus on a function $f$ with a single critical point to see how the behavior of the spectrum depends on the behavior of $f$ near the critical point. Specifically, we will consider

$$
f(x)=\frac{1}{1+|x|^{\kappa}}
$$

for arbitrary $\kappa>0$. The main result of this paper is

Theorem 1.2. Fix $\kappa>0$ and let $f(x)$ be as in (1). Then there is a positive constant $C_{\kappa}$ such that all eigenvalues of $L_{\gamma}$ lie in the half plane

$$
\left\{z: \operatorname{Re} z \geq C_{\kappa}|\gamma|^{2 /(\kappa+2)}\right\} \text {. }
$$

Remarks. (1) Since the spectrum $\sigma\left(L_{\gamma}\right)$ consists only of eigenvalues, the theorem is succinctly stated as the inclusion $\sigma\left(L_{\gamma}\right) \subset\left\{z: \operatorname{Re}(z) \geq C|\gamma|^{2 /(\kappa+2)}\right\}$. (2) The proof relies on a quadratic form estimate which only gives lower bounds. However, in [9] it is mentioned that numerical calculations by Thiery Gallay for $\kappa=2$ suggest that the obtained rate $\gamma^{1 / 2}$ is sharp. This is to be contrasted with the rate $\gamma^{1 / 4}$ provided by the commutator methods of Villani [9].

A recent paper of Gallagher et al. [4] gives a detailed study of the so-called pseudospectrum of $L_{\gamma}$ for a somewhat different class of perturbations $f$. Specifically, they consider the "pseudspectral quantity" $\Psi(\gamma)=1 /\left(\sup _{\lambda \in \mathbb{R}}\left\|\left(L_{\gamma}-\mathrm{i} \lambda\right)^{-1}\right\|\right)$ and ask how it diverges as $\gamma \rightarrow \infty$. They obtain a sufficient condition for divergence of $\Psi(\gamma)$ that is very similar to Theorem 1.1, but which they prove directly without noting the connection to the work of Constanin et al. [1]. By placing various conditions on critical points of $g$ they obtain a result similar to Theorem 1.2 although phrased as a bound on $\Psi(\gamma)$ rather than the low lying eigenvalues of $L_{\gamma}$.

In $[4,9]$, this problem is motivated as a simplified version of a spectral problem that arises in the linear stability analysis of a $2 \mathrm{D}$ hydrodynamic equation. In that context, one is mainly interested in understanding the $t \rightarrow \infty$ asymptotic behavior of solutions to the initial value problem

$$
\partial_{t} \psi(x, t)=\partial_{x}^{2} \psi(x, t)-x^{2} \psi(x, t)-\mathrm{i} \gamma \frac{1}{1+|x|^{\kappa}} \psi(x, t), \quad \psi(x, t)=\psi_{0}(x),
$$


The semi-group $S_{t}=\mathrm{e}^{-t L_{\gamma}}$ generates solutions to $(2)$ via $\psi(x, t)=S_{t} \psi_{0}(x)$. It follows that the large $t$ asymptotic behavior is governed by the eigenvalue of $L_{\gamma}$ with smallest real part. Indeed,

$$
\left\|\mathrm{e}^{-t L_{\gamma}}\right\| \leq C_{\gamma, \alpha} \mathrm{e}^{-\alpha t}
$$

for any $\alpha<\inf \operatorname{Re}\left(\sigma\left(L_{\gamma}\right)\right)$, where for $A \subset \mathbb{C}$ we let $\operatorname{Re}(A)=\{\operatorname{Re}(z): z \in A\}$. Hence, the conclusion of the theorem implies that

$$
\int_{\mathbb{R}}|\psi(x, t)|^{2} \mathrm{~d} x \leq C_{\gamma} \mathrm{e}^{-C \gamma^{2 /(\kappa+2)} t},
$$

for solutions to (2).

The estimate (3) is quite striking as in some sense the convergence of $\psi \rightarrow 0$ is driven entirely by the "dissipative" term $\partial_{x}^{2} \psi-x^{2} \psi$ on the right. Indeed, without the dissipative term, the solution would be

$$
\psi(x, t)=\mathrm{e}^{-\mathrm{i} \gamma \frac{t}{1+|x|^{\kappa}}} \psi_{0}(x),
$$

which has constant $L^{2}$ norm. If the $L^{2}$ conserving term $-\mathrm{i} \gamma /\left(1+|x|^{\kappa}\right) \psi$ is absent then the $L^{2}$ norm goes to zero, but at the modest rate $\mathrm{e}^{-t}$ given by finding the smallest eigenvalue of $L_{0}$. However the combination of the "conservative" and "dissipative" terms results in much faster convergence to zero. This phenomenon has been dubbed "hypocoercivity" by Villani, in analogy with Hörmander's notion of "hypoelipticity."

From another point of view, this is not so surprising. After all, those of us who add milk to our tea know to stir the cup of tea after adding milk to speed up the mixing of milk and tea. More or less this is the same phenomenon as what is described in Equation 2. After all, on its own the milk would eventually diffuse through the cup of tea. However, we may greatly decrease the time to achieve diffusion by stirring, which essentially adds a convective term to the diffusion equation for the density of tea. This convection on it's own, in an imaginary liquid with no dissipation, would only set the milk and tea in ever circulating motion - it is "conservative!" Together the dissipative and conservative motions combine into a flow which mixes much faster.

In recent years the mathematical analysis of hypocoercivity and related problems has been the subject of much research. Constantin et al. [1] have analyzed the phenomenon from an abstract functional analytic perspective summarized in the following theorem:

Theorem 1.3 (Theorem 11.4 of [1]). Let $H_{0}$ be a self-adjoint, positive, unbounded operator with discrete spectrum and let $F$ be self-adjoint and satisfy

$$
\|F \psi\|^{2} \leq C\left\langle\psi, H_{0} \psi\right\rangle \text { and }\left\langle\mathrm{e}^{\mathrm{i} F t} \psi, H_{0} \mathrm{e}^{\mathrm{i} F t} \psi\right\rangle \leq B(t)\left\langle\psi, H_{0} \psi\right\rangle
$$

with constants $C$ and $B(t)$ independent of $\psi$ and $B(t) \in L_{\mathrm{loc}}^{2}(0, \infty)$. Then for any $\gamma>0$ the operator $H_{0}+\mathrm{i} \gamma F$ generates a contractive semi-group and the following are equivalent

- For any $t>0$

$$
\lim _{\gamma \rightarrow \infty}\left\|\mathrm{e}^{-t\left(H_{0}+\mathrm{i} \gamma F\right)}\right\|=0
$$

- The operator $F$ has no eigenvectors in $\mathcal{Q}\left(H_{0}\right)=$ form domain of $H_{0}$. 
Using Theorem 1.3 we may easily prove Theorem 1.1 . Note that any normalized eigenvector $\psi$ satisfies

$$
\left\|\mathrm{e}^{-t\left(H_{0}+\mathrm{i} \gamma F\right)} \psi\right\|=\mathrm{e}^{-t \operatorname{Re} \lambda}
$$

with $\lambda$ the corresponding eigenvalue. It follows that all eigenvalues satisfy:

$$
\operatorname{Re} \lambda \geq-\frac{1}{t} \ln \left\|\mathrm{e}^{-t\left(H_{0}+\mathrm{i} \gamma F\right)}\right\| .
$$

Thus (4) implies that $\lim _{\gamma \rightarrow \infty} \inf \operatorname{Re} \sigma\left(H_{0}+\gamma F\right)=\infty$. This result applies in the present context, with the operator $H_{0}=-\partial_{x}^{2}+x^{2}$ and $F=f(x)$. The form domain of $H_{0}$ is the space

$$
\mathcal{H}_{1}=\left\{\psi: \partial_{x} \psi \text { and } x \psi \in L^{2}(\mathbb{R})\right\}
$$

An eigenvector of $F$ is a function supported on a level set of $f$. Thus $\left\|\mathrm{e}^{-t\left(H_{0}+\mathrm{i} \gamma F\right)}\right\| \rightarrow$ 0 as $\gamma \rightarrow \infty$ if and only if the level sets of $f$ support no (non-trivial) $\mathcal{H}_{1}$ eigenfunctions. Since $\mathcal{H}_{1} \subset C(\mathbb{R})$ Theorem 1.1 follows.

However, (5) and (4) do not provide quantitative information on the rate of divergence. To find this we must estimate, for finite $\gamma$, the location of the eigenvalues. Before proceeding, it is instructive to ask, in general, "how do we estimate the location of spectrum?" When one is available, a variational principle relating eigenvalues to extrema of a quadratic form is one of the most effective tools. For example, consider the self-adjoint eigenvalue problem

$$
-\partial_{x}^{2} \psi(x)+V(x) \psi(x)=\lambda \psi(x),
$$

with real valued $V$ that diverges to $+\infty$ as $x \rightarrow \pm \infty$. The smallest $\lambda \in \mathbb{R}$ such that a solution $\psi \in L^{2}(\mathbb{R})$ may be found is called the ground state eigenvalue and satisfies a variational principle

$$
\lambda_{0}=\inf \left\{\int_{-\infty}^{\infty}\left|\partial_{x} \psi(x)\right|^{2}+\left.V(x)|\psi(x)|^{2} d x\left|\int_{-\infty}^{\infty}\right| \psi(x)\right|^{2} d x=1\right\} .
$$

Furthermore a minimizing wave function $\psi$ satisfies the eigenvalue Equation (7). The min-max principle generalizes this to higher eigenvalues.

It would be hard to overstate the utility of (8). To find an upper bound on $\lambda_{0}$ we just compute the energy $\mathcal{E}(\psi)=\int_{-\infty}^{\infty}\left|\partial_{x} \psi(x)\right|^{2}+V(x)|\psi(x)|^{2} d x$ of any normalized function $\psi \in L^{2}(\mathbb{R})$. To find a good upper bound we "simply" make a suitably clever choice of $\psi$. Finding a lower bound is often more involved, requiring a uniform estimate from below on $\mathcal{E}(\psi)$, but even that is often possible, particularly in an asymptotic limit (e.g., in the semi-classical limit $[5,6,8]$ ). In way of contrast, an exact analysis of the eigenvalue problem (7) is possible only for a few explicitly solvable examples.

However, for the operators $L_{\gamma}$ considered here — which are not self-adjoint - a direct variational approach provides little insight. It remains true that any eigenvalue $\lambda$ satisfies

$$
\operatorname{Re} \lambda \geq \inf \left\{\operatorname{Re}\left\langle\psi, L_{\gamma} \psi\right\rangle: \int|\psi(x)|^{2}=1\right\},
$$

since $\operatorname{Re} \lambda=\operatorname{Re}\left\langle\psi_{\lambda}, L_{\gamma} \psi_{\lambda}\right\rangle$ with $\psi_{\lambda}$ the corresponding normalized eigenfunction. However, the infimum on the right-hand side (r.h.s.) is insensitive to the imaginary 
term $\mathrm{i} \gamma /\left(1+|x|^{\kappa}\right)^{m}$ in the operator, and thus is independent of $\gamma$. So all we learn is that

$$
\operatorname{Re} \lambda \geq \inf \left\{\operatorname{Re}\left\langle\psi, L_{0} \psi\right\rangle: \int|\psi(x)|^{2}=1\right\}=1,
$$

using the well known explicit diagonalization of $L_{0}=-\partial_{x}^{2}+x^{2}$.

The cornerstone of the variational approach is a relation between numerical range and spectrum valid only for normal operators. ${ }^{1}$ The numerical range of a linear operator $L$ on a Hilbert space $\mathcal{H}$ refers to the set

$$
\operatorname{num}(L)=\left\{\langle\psi, L \psi\rangle_{\mathcal{H}} \mid \psi \in \mathcal{H} \text { and }\|\psi\|_{\mathcal{H}}=1\right\}
$$

If $L$ is normal then we have

$$
\operatorname{cch}(\sigma(L))=\operatorname{cl}(\operatorname{num}(L))
$$

where cch denotes the "closed convex hull" and cl denotes topological closure. This may be seen using the spectral theorem, since it is elementary for multiplication operators on $L^{2}$ spaces. In particular, one has the following variational principle for normal operators: extreme points of the closed numerical range are in the spectrum.

For a general closed operator $L$ we do not have (10). However, since the point spectrum of an operator $L$ clearly falls in $\operatorname{num}(L)$ we have

$$
\sigma(L) \subset \operatorname{cl}(\operatorname{num}(L))
$$

whenever $L$ has compact resolvent - as do the operators $L_{\gamma}$ considered here. ${ }^{2}$ In fact, since $\operatorname{num}(L)$ is convex [7, Theorem V.3.1], we have

$$
\operatorname{cch}(\sigma(L)) \subset \operatorname{cl}(\operatorname{num}(L))
$$

for $L$ with compact resolvent. However, there does not appear to be a relation between spectrum and numerical range valid for all operators $L$ with compact resolvent beyond the inclusion (12). Furthermore one knows from examples that the spectrum may lie arbitrarily far from the extreme points of the numerical range. For example, the numerical range of a $2 \times 2$ matrix is an ellipse with foci at the eigenvalues, but the radii of the ellipse may be arbitrarily large.

One quite natural idea is to exploit the invariance of $\sigma(L)$ under the map $L \mapsto$ $T^{-1} L T$ where $T$ is bounded with bounded inverse. This map fixes the numerical range only if $T$ is unitary, so we may gain something by this procedure. Indeed, if we were lucky enough to find $T$ so that $T^{-1} L T$ were normal, we would have a variational principle for the eigenvalues of $L$ using the quadratic form $\left\langle\psi, T^{-1} L T \psi\right\rangle$. Even if $T^{-1} L T$ is not normal, we may hope to use $T$ to bring the extreme points of the numerical range closer to the spectrum of $L$. In finite dimension, this procedure will work, in principle, for any $L$. Indeed, if $\operatorname{dim}(\mathcal{H})<\infty$ then

$$
\operatorname{cch}(\sigma(L))=\bigcap_{T \in \mathcal{G} \mathcal{L}(\mathcal{H})} \operatorname{cl}\left(\operatorname{num}\left(T^{-1} L T\right)\right) .
$$

\footnotetext{
${ }^{1}$ Recall that $L$ is normal if it commutes with its adjoint $L^{\dagger}$. Lack of self-adjointness is not really the problem here. The variational approach would work in principle if $L_{\gamma}$ were normal.

${ }^{2}$ Equation (11) also holds whenever $L$ is bounded, but may fail for an unbounded closed operator. For example consider $M \psi=\psi^{\prime}$ on the space $H_{0}^{1}(0,1)$ of functions vanishing at 0 and 1 but with $L^{2}$ derivative. Then $M$ is closed and $\operatorname{num}(M)=\mathrm{i} \mathbb{R}$, but $\sigma(M)=\mathbb{C} \operatorname{since} \operatorname{ran}(M-z I)$ is perpendicular to $e^{\bar{z} x}$ for any $z \in \mathbb{C}$.
} 
In fact, using the Jordan canonical form one can show a bit more: for any $\epsilon>0$ there is $T_{\epsilon} \in \mathcal{G} \mathcal{L}(\mathcal{H})$ such that $\operatorname{cl}\left(\operatorname{num}\left(T_{\epsilon}^{-1} L T_{\epsilon}\right)\right)$ is contained in the $\epsilon$ neighborhood of $\operatorname{cch}(\sigma(L))$. It is not clear if (13) holds for general $L$ with compact resolvent when $\operatorname{dim}(\mathcal{H})=\infty$. Anyway the answer to this question may not be so relevant, as in practice it is rather difficult to produce an effective conjugating operator without already knowing the Jordan form of $L$.

So how do we locate the spectrum of $L_{\gamma}$ ? Let us first consider an heuristic approach, that is very close in spirit to the rigorous method applied below. Let $\lambda$ be an eigenvalue, $L_{\gamma} \psi=\lambda \psi$, with eigenfunction $\psi$. Suppose that $\psi$ has a holomorphic extension $\psi(z)$ for $z \in C_{\beta}:=\left\{x \mathrm{e}^{\mathrm{i} \theta} \quad: x \in \mathbb{R}\right.$ and $\left.|\theta|<\beta\right\}$. By rotating the contour on which we evaluate the eigenfunction equation we find that

$$
-\mathrm{e}^{-2 \mathrm{i} \theta} \partial_{x}^{2} \phi(x)+\mathrm{e}^{2 \mathrm{i} \theta} x^{2} \phi(x)+\mathrm{i} \gamma \frac{1}{1+\mathrm{e}^{\kappa \mathrm{i} \theta}|x|^{\kappa}} \phi(x)=\lambda \phi(x),
$$

where $\phi(x)=\psi\left(\mathrm{e}^{\mathrm{i} \theta} x\right)$ for some $\theta<\beta$. (We have extended $|x|^{\kappa}$ to the holomorphic function $\left(z^{2}\right)^{\frac{\kappa}{2}}$, single valued on $C_{\beta}$ with a branch singularity at 0 provided $\beta$ is not too large.) Assuming the complex rotated function $\phi$ is square integrable, we discover that $\lambda$ is an eigenvalue of

$$
L_{\gamma}^{(\mathrm{i} \theta)}=-\mathrm{e}^{-2 \mathrm{i} \theta} \partial_{x}^{2}+\mathrm{e}^{2 \mathrm{i} \theta} x^{2}+\mathrm{i} \gamma \frac{1}{1+\mathrm{e}^{\kappa \mathrm{i} \theta}|x|^{\kappa}} .
$$

In particular, applying (9) with $L_{\gamma}^{(\mathrm{i} \theta)}$ in place of $L_{\gamma}$, we find $\operatorname{Re} \lambda \geq \inf \sigma\left(\operatorname{Re} L_{\gamma}^{(\mathrm{i} \theta)}\right)$ where

$$
\operatorname{Re} L_{\gamma}^{(\mathrm{i} \theta)}=\frac{1}{2}\left[L_{\gamma}^{(\mathrm{i} \theta)}+L_{\gamma}^{(\mathrm{i} \theta)^{\dagger}}\right]=\cos (2 \theta)\left\{-\partial_{x}^{2}+x^{2}+\gamma \frac{\sin (\kappa \theta)}{\cos (2 \theta)} \frac{|x|^{\kappa}}{\left.\left.\left|1+\mathrm{e}^{\kappa \mathrm{i} \theta}\right| x\right|^{\kappa}\right|^{2}}\right\} .
$$

The operator in curly brackets is a Schrödinger operator with (real valued) potential

$$
V(x)=x^{2}+\gamma \frac{\sin (\kappa \theta)}{\cos (2 \theta)} \frac{|x|^{\kappa}}{\left.\left.\left|1+\mathrm{e}^{\kappa \mathrm{i} \theta}\right| x\right|^{\kappa}\right|^{2}} .
$$

If $\theta$ is sufficiently small then $V(x)>x^{2}$ and near the origin

$$
V(x) \sim x^{2}+\gamma \frac{\sin (\kappa \theta)}{\cos (2 \theta)}|x|^{\kappa}
$$

Applying ideas from semi-classical analysis, as in $[5,6,8]$, one concludes for large $\gamma$ that the ground state of $\operatorname{Re} L_{\gamma}^{(\mathrm{i} \theta)}$ is an approximate ground state of

$$
\cos (2 \theta)\left\{-\partial_{x}^{2}+\gamma \frac{\sin (\kappa \theta)}{\cos (2 \theta)}|x|^{\kappa}\right\}
$$

whose ground state eigenvalue is seen to be proportional to $\gamma^{2 /(\kappa+2)}$ by scaling.

There are two deficiencies with the above argument. First we do not know that the wave function is analytic. Second, even if $\psi$ were analytic, there would be no a priori reason to believe that $\phi \in L^{2}$. A reader familiar with the technique of "complex scaling" from scattering theory for (self-adjoint) Schrödinger operators will recognize 
the way out. We simply ignore these problems, focusing our attention instead on the spectral analysis of the following analytic family of operators:

$$
L_{\gamma}^{(w)}=-\mathrm{e}^{-2 w} \partial_{x}^{2}+\mathrm{e}^{2 w} x^{2}+\mathrm{i} \gamma \frac{1}{1+\mathrm{e}^{\kappa w}|x|^{\kappa}}
$$

with $w=u+\mathrm{i} \theta$ a complex parameter in a strip $\{|\theta|<\delta\}$ with $\delta$ sufficiently small. For real $w=u$, with $\theta=0$, we have

$$
L_{\gamma}^{(u)}=T_{u} L_{\gamma} T_{u}^{\dagger}
$$

where $T_{u}$ is the unitary dilation operator,

$$
T_{u} \psi(x)=\mathrm{e}^{\frac{1}{2} u} \psi\left(\mathrm{e}^{u x}\right) .
$$

However, for complex $w, L_{\gamma}^{(w)}$ is not obtained from $L_{\gamma}$ by conjugation, but rather by analytic continuation from the operator valued map $u \mapsto L_{\gamma}^{(u)}$. With this set-up, we will prove Theorem 1.2 in two steps:

(1) From analyticity we will show that the spectrum of $L_{\gamma}^{(w)}$ is independent of $w$ as $w$ varies in the strip $\{|\operatorname{Im} w|<\delta\}$.

(2) Using semi-classical analysis of $\operatorname{Re} L_{\gamma}^{(i \theta)}$ for $\theta \neq 0$ we will obtain an effective estimate on the real part of the lowest eigenvalue.

\section{Proof}

For each $\alpha>0$ let $S_{\alpha}=\{w \in \mathbb{C}:|\operatorname{Im} w|<\alpha\}$. The first step of the argument is to show that $w \mapsto\left[L_{\gamma}^{(w)}\right]^{-1}$ is a compact operator valued analytic map on a strip $S_{\alpha_{\kappa}}$.

\section{Lemma 2.1. Let}

$$
\alpha_{\kappa}=\pi \min \left(\frac{1}{2 \kappa}, \frac{1}{2}\right) .
$$

For each $w \in S_{\alpha_{\kappa}}$ the operator $L_{\gamma}^{(w)}$ has compact resolvent and

$$
\sigma\left(L_{\gamma}^{(w)}\right)=\sigma\left(L_{\gamma}\right)
$$

Proof. We will analyze $L_{\gamma}^{(w)}$ through the associated quadratic forms

$$
Q_{\gamma}^{(w)}(\psi)=\mathrm{e}^{-2 w}\left\|\partial_{x} \psi\right\|_{L^{2}}^{2}+\mathrm{e}^{2 w}\|x \psi\|_{L^{2}}^{2}+\mathrm{i} \alpha \int_{-\infty}^{\infty} \frac{1}{1+\mathrm{e}^{\kappa w}|x|^{\kappa}}|\psi(x)|^{2} d x,
$$

defined on the form domain $\mathcal{H}_{1}$ (see (6)). Our goal is to show, in the terminology of Kato [7], that $w \mapsto Q_{\gamma}^{(w)}$ is a holomorphic family of type (a), which is to say

(1) $w \mapsto Q_{\gamma}^{(w)}(\psi)$ is a holomorphic map for each $\psi \in \mathcal{H}_{1}$, and

(2) for fixed $w$ the form $Q_{\gamma}^{(w)}(\cdot)$ is sectorial and closed on $\mathcal{H}_{1}$.

Once we have shown (1) and (2), it follows by definition that $L_{\gamma}^{(w)}$ is a "holomorphic family of type (B)" and thus, by Theorem 4.3 of [7] that $L_{\gamma}^{(w)}$ either has compact resolvent for all $w$ or for no $w$. Since $L_{\gamma}=L_{\gamma}^{(0)}$ has compact resolvent it follows that $L_{\gamma}^{(w)}$ has compact resolvent for all $w$. 
Take (1) and (2) for granted for the moment. Then $w \mapsto L_{\gamma}^{(w)}$ is a holomorphic family with compact resolvent. By [7, Theorem VII.1.9], we have the further dichotomy that $\lambda \in \mathbb{C}$ is an eigenvalue of $L_{\gamma}^{(w)}$ either for $w$ in a discrete set or for all $w \in S_{\alpha_{k}}$. We may rule out the possibility of a discrete set by noting that

$$
L_{\gamma}^{(u+\mathrm{i} \theta)}=T_{u}^{\dagger} L_{\gamma}^{(\mathrm{i} \theta)} T_{u}
$$

where $T_{u}$ is the unitary dilation operator (16). That is, the family $u \mapsto L^{(u+\mathrm{i} \theta)}$ is related by unitary conjugations and is thus isospectral. Thus $L_{\gamma}^{(w)}$ is isospectral as claimed and the lemma is proved once we verify (1) and (2).

Turning now to (1) and (2), note that (1) follows easily from the explicit expression for $Q_{\gamma}^{(w)}$. (Since Re $\mathrm{e}^{\kappa w}>0$ on $S_{\alpha_{\kappa}}$ the integrand in the third term on the r.h.s. of (17) cannot develop a singularity.) To prove (2), namely sectoriality, note that

$$
Q_{\gamma}^{(u+\mathrm{i} \theta)}=Q_{\gamma}^{(\mathrm{i} \theta)} \circ T_{u}
$$

where the dilation $T_{u}$ clearly maps $\mathcal{H}_{1}$ onto $\mathcal{H}_{1}$. Thus $Q_{\gamma}^{(u+\mathrm{i} \theta)}$ is closed and sectorial if and only if $Q_{\gamma}^{(\mathrm{i} \theta)}$ is closed and sectorial.

To show that $Q_{\gamma}^{(\mathrm{i} \theta)}$ is closed and sectorial, note that

$$
\operatorname{Re} Q_{\gamma}^{(\mathrm{i} \theta)}(\psi)=\cos (2 \theta)\left\{\left\|\partial_{x} \psi\right\|_{L^{2}}^{2}+\|x \psi\|_{L^{2}}^{2}\right\}-\gamma \int_{-\infty}^{\infty} \operatorname{Im} \frac{1}{1+\mathrm{e}^{\mathrm{i} \kappa \theta}|x|^{\kappa}}|\psi(x)|^{2} d x
$$

Thus, since $\cos (\kappa \theta)>0$ for $|\theta|<\alpha_{\kappa}$, we have

$$
\cos (2 \theta) Q_{0}(\psi)-|\gamma|\|\psi\|_{L^{2}}^{2} \leq \operatorname{Re} Q_{\gamma}^{(\mathrm{i} \theta)}(\psi) \leq \cos (2 \theta) Q_{0}(\psi)+|\gamma|\|\psi\|_{L^{2}}^{2},
$$

where

$$
Q_{0}(\psi)=\left\|\partial_{x} \psi\right\|_{L^{2}}^{2}+\|x \psi\|_{L^{2}}^{2}
$$

Now

$$
\operatorname{Im} Q_{\gamma}^{(\mathrm{i} \theta)}(\psi)=\sin (2 \theta)\left\{-\left\|\partial_{x} \psi\right\|_{L^{2}}^{2}+\|x \psi\|_{L^{2}}^{2}\right\}+\gamma \int_{-\infty}^{\infty} \operatorname{Re} \frac{1}{1+\mathrm{e}^{\mathrm{i} \kappa \theta}|x|^{\kappa}}|\psi(x)|^{2} d x .
$$

Thus, by (18),

$$
\begin{aligned}
\left|\operatorname{Im} Q_{\gamma}^{(\mathrm{i} \theta)}(\psi)\right| \leq \sin (2 \theta) Q_{0}(\psi) & +|\gamma|\|\psi\|_{L^{2}}^{2} \\
& \leq \tan (2 \theta) \operatorname{Re} Q_{\gamma}^{(\mathrm{i} \theta)}(\psi)+|\gamma|\left(1+\frac{1}{\cos (2 \theta)}\right)\|\psi\|_{L^{2}}^{2},
\end{aligned}
$$

which is to say $Q_{\gamma}^{(\mathrm{i} \theta)}$ is sectorial. Furthermore, since $Q_{0}$ is a closed form with domain $\mathcal{H}_{1}$ it follows from $(18)$ that $\operatorname{Re} Q_{\gamma}^{(\mathrm{i} \theta)}$ and hence $Q_{\gamma}^{(\mathrm{i} \theta)}$ is closed on $\mathcal{H}_{1}$.

2.1. Analysis of $\operatorname{Re} L_{\gamma}^{(\mathrm{i} \theta)}$. Since $L_{\gamma}$ and $L_{\gamma}^{(\mathrm{i} \theta)}$ are isospectral, if $\lambda$ is an eigenvalue of $L_{\gamma}$ then

$$
\operatorname{Re} \lambda \geq \cos (2 \theta) \min \sigma\left(H_{\gamma}(\theta)\right)
$$

where $H_{\gamma}(\theta)$ is the real part of $\frac{1}{\cos (2 \theta)} L_{\gamma}^{(\mathrm{i} \theta)}$. That is,

$$
H_{\gamma}(\theta)=-\partial_{x}^{2}+x^{2}+\gamma \frac{\sin (\kappa \theta)}{\cos (2 \theta)} \frac{|x|^{\kappa}}{\left.\left.\left|1+\mathrm{e}^{\mathrm{i} \kappa \theta}\right| x\right|^{\kappa}\right|^{2}} .
$$


Note that $H_{\gamma}(\theta)=H_{-\gamma}(-\theta)$. Hence without loss of generality we may take $\gamma>0$. For the rest of the proof, let us fix some $\theta \in\left(0, \alpha_{\kappa}\right)$. Theorem 1.2 will follow once we show that the ground state eigenvalue $\lambda_{0}=\lambda_{0}(\theta)$ of $H_{\gamma}(\theta)$ satisfies an estimate

$$
\lambda_{0} \geq C \gamma^{\frac{2}{\kappa+2}}
$$

(In the end one could try to optimize over the choice of $\theta$, however this would affect only the proportionally constant not the rate of divergence of the eigenvalue.)

The Schrödinger operator $H_{\gamma}(\theta)$ is of the form

$$
-\partial_{x}^{2}+V(x)
$$

where

$$
V(x)=x^{2}+\gamma \frac{\sin (\kappa \theta)}{\cos (2 \theta)} \frac{|x|^{\kappa}}{\left.\left.\left|1+\mathrm{e}^{\mathrm{i} \kappa \theta}\right| x\right|^{\kappa}\right|^{2}} .
$$

Thus $V(x)$ has a global minimum at $x=0$, with

$$
V(x)=\gamma \frac{\sin (k \theta)}{\cos (2 \theta)}|x|^{\kappa}+x^{2}+o\left(|x|^{\kappa}\right), \quad|x| \rightarrow 0 .
$$

Equation (19) follows if we can show that the ground state eigenvalue of $H_{\gamma}(\theta)$ is asymptotic to the ground state eigenvalue of the anharmonic oscillator

$$
K_{\alpha}=-\partial_{x}^{2}+\alpha|x|^{\kappa}
$$

with $\alpha=\gamma \frac{\sin (k \theta)}{\cos (2 \theta)}$. Indeed, scaling shows that the ground state of $K_{\alpha}$ satisfies $\lambda_{0}\left(K_{\alpha}\right)=\lambda_{0}\left(K_{1}\right) \alpha^{\frac{2}{2+\kappa}}$.

Let $\Phi$ be a fixed $C^{2}$ "cut-off" function, with

(1) $0 \leq \Phi(x) \leq 1$ for all $x$,

(2) $\Phi(x)=1$ for $|x| \leq 1$,

(3) $\Phi(x)=0$ for $|x| \geq 2$.

Fix $\nu>0$, to be chosen below, and let

$$
J(x)=\Phi\left(\alpha^{\nu} x\right), \quad \text { with } \alpha=\gamma \frac{\sin (k \theta)}{\cos (2 \theta)} .
$$

Define $\widetilde{J}(x)$ so that $\widetilde{J}(x)^{2}+J(x)^{2}=1$, that is $\widetilde{J}(x)=\sqrt{1-J(x)^{2}}$. By the IMS localization formula (see [2, Theorem 3.2]),

$$
H_{\gamma}(\theta)=\widetilde{J} H_{\gamma}(\theta) \widetilde{J}+J H_{\gamma}(\theta) J-(\partial \widetilde{J})^{2}-(\partial J)^{2} .
$$

We will estimate each of the four operators on the r.h.s. of (21) separately. To estimate $\widetilde{J} H(\theta) \widetilde{J}$, we simply drop the kinetic term $-\partial_{x}^{2}$, to obtain

$$
\widetilde{J} H_{\gamma}(\theta) \widetilde{J} \geq \inf _{|x|>\alpha^{-\nu}}\left[x^{2}+\alpha \frac{|x|^{\kappa}}{\left.\left.\left|1+\mathrm{e}^{\mathrm{i} \kappa \theta}\right| x\right|^{\kappa}\right|^{2}}\right] \widetilde{J}^{2} \geq \inf _{y>\alpha^{-\kappa \nu}}\left[y^{2 / \kappa}+\alpha \frac{y}{(1+y)^{2}}\right] \widetilde{J}^{2} .
$$

To minimize $g(y)=y^{2 / \kappa}+\alpha \frac{y}{(1+y)^{2}}$ over $y>\alpha^{-\kappa \nu}$ we must compare

$$
g\left(\alpha^{-\kappa \nu}\right)=\alpha^{-2 \nu}+\alpha \frac{\alpha^{-\kappa \nu}}{\left(1+\alpha^{-\kappa \nu}\right)^{2}} \sim C \alpha^{1-\kappa \nu}
$$

with the value of $g$ at the stationary points, which solve

$$
\frac{2}{\kappa \alpha} y^{\frac{2}{\kappa}-1}(1+y)^{3}+1=y
$$


There are two solutions to this equation, $y_{1} \sim 1$ and $y_{2} \sim C \alpha^{\frac{\kappa}{2+\kappa}}$ as $\alpha \rightarrow \infty$. Evaluating $g$ at these points, we obtain

$$
g\left(y_{1}\right) \sim \frac{1}{2} \alpha \text { and } g\left(y_{2}\right) \sim C \alpha^{\frac{2}{2+\kappa}}
$$

Thus, provided we take $\nu \leq \frac{2}{2+\kappa}$, we conclude that

$$
\widetilde{J} H_{\gamma}(\theta) \widetilde{J} \geq C \alpha^{\frac{2}{2+\kappa}} \widetilde{J}^{2}
$$

as $\alpha \rightarrow \infty$.

To estimate $J H_{\gamma}(\theta) J$ we compare to the an harmonic oscillator (20):

$$
\begin{aligned}
J H_{\gamma}(\theta) J-J K_{\alpha} J & =\left[x^{2}+\alpha|x|^{\kappa}\left[\frac{1}{\left.\left.\left|1+\mathrm{e}^{\mathrm{i} \kappa \theta}\right| x\right|^{\kappa}\right|^{2}}-1\right]\right] J^{2} \\
& \geq-\alpha \sup _{|x| \leq 2 \alpha^{-\nu}}\left[|x|^{\kappa}\left[1-\frac{1}{\left.\left.|1+| x\right|^{\kappa}\right|^{2}}\right]\right] J^{2} \sim-C \alpha^{1-2 \kappa \nu} J^{2},
\end{aligned}
$$

as $\alpha \rightarrow \infty$. Putting this together with the estimate for $\widetilde{J} H_{\gamma}(\theta) \widetilde{\gamma}$ and the estimate $K_{\alpha} \geq C \alpha^{\frac{2}{2+\kappa}}$, we see that

$$
\begin{aligned}
H_{\gamma}(\theta) & =J K_{\alpha} J+J\left(H_{\gamma}(\theta)-K_{\alpha}\right) J+\widetilde{J} H_{\gamma}(\theta) \widetilde{J}-\left(\partial_{x} J\right)^{2}-\left(\partial_{x} \widetilde{J}\right)^{2} \\
& \geq C \alpha^{\frac{2}{2+\kappa}} J^{2}+C \alpha^{1-2 \kappa \nu} J^{2}+C \alpha^{\frac{2}{2+\kappa}} \widetilde{J}^{2}-C \alpha^{2 \nu} \\
& \geq C \alpha^{\frac{2}{2+\kappa}}-\left\{C \alpha^{1-2 \kappa \nu} J^{2}+C \alpha^{2 \nu}\right\}
\end{aligned}
$$

since $\left|\partial_{x} J\right|,\left|\partial_{x} \widetilde{J}\right| \leq C \alpha^{\nu}$. To minimize the growth of the error term (in brackets), let us choose $\nu$ so that the two terms are of equal magnitude, namely $\nu=\frac{1}{2+2 \kappa}$, yielding

$$
H_{\gamma}(\theta) \geq C \gamma^{\frac{2}{2+\kappa}}-C \gamma^{\frac{1}{1+\kappa}}
$$

and completing the proof.

\section{Acknowledgments}

I would like to express my gratitude for the hospitality of Tom Spencer and the Institute for Advanced Study, where I was a member from 2005-2007. I learned of the problem considered here from Cedric Villani during a very pleasant discussion with him and Tom at the IAS in 2007. I benefited also from discussions with Andrej Zlatos, who pointed out the relevance of Theorem 1.4 of [1]. The final version of this manuscript was prepared at the Centre Interfacultaire Bernoulli of the Ecole Polytechnic Federal de Lausanne during a visit in connection with the program Spectral and Dynamical Properties of Quantum Hamiltonians. This work supported in part by NSF CAREER Award DMS-08446325.

\section{References}

[1] P. Constantin, A. Kiselev, L. Ryzhik and A. Zlatoš, Diffusion and mixing in fluid flow, Ann. Math. (2) 168(2) (2008), 643-674.

[2] H. L. Cycon, R. G. Froese, W. Kirsch and B. Simon, Schrödinger operators with application to quantum mechanics and global geometry, Texts and Monographs in Physics, Springer-Verlag, Berlin, 1987. 
[3] B. Franke, C.-R. Hwang, H.-M. Pai and S.-J. Sheu, The behavior of the spectral gap under growing drift, Trans. Amer. Math. Soc. 362(03) (2009), 1325-1350.

[4] I. Gallagher, T. Gallay and F. Nier, Spectral asymptotics for large skew-symmetric perturbations of the harmonic oscillator, Int. Math. Res. Not. 2009(12) (2009), 2147-2199.

[5] B. Helffer and J. Sjostrand, Multiple wells in the semiclassical limit. I, Comm. Partial Differential Equations 9(4) (1984), 337-408.

[6] B. Helffer and J. Sjostrand, Puits multiples en limite semi-classique. II. Interaction moléculaire. Symétries. Perturbation, Annales de l'Institut Henri Poincaré. Physique Théorique 42(2) (1985), $127-212$.

[7] T. Kato, Perturbation theory for linear operators, Classics in Mathematics, Springer-Verlag, Berlin, 1995.

[8] B. Simon, Semiclassical analysis of low lying eigenvalues. I. Nondegenerate minima: asymptotic expansions, Annales de l'Institut Henri Poincaré 38(4) (1983), 309-347.

[9] C. Villani, Hypocoercive diffusion operators, in International Congress of Mathematicians, III, European Mathematical Society, Zürich, 2006, 473-498.

[10] A. Zlatoš, Private Communication, 2010.

Department of Mathematics, Michigan State University, East Lansing, Mi 48823, USA E-mail address: jeffrey@math.msu.edu

$U R L:$ http://www.math.msu.edu/jeffrey 
